\title{
Diagnostic Value of Ultrasound and Gastrointestinal Series Findings in Detection of Pediatric Intestinal Malrotation
}

\author{
Mina Taghavi, ${ }^{1}$ Seyed Ali Alamdaran, ${ }^{1,}$ and Ali Feizi ${ }^{1}$ \\ ${ }^{1}$ Department of Radiology, Faculty of Medicine, Mashhad University of Medical Sciences, Mashhad, Iran \\ "Corresponding author: Seyed Ali Alamdaran M.D., Department of Radiology, Imam Reza Hospital, Faculty of Medicine, Mashhad University of Medical Sciences, Mashhad, Iran. \\ Tel: +98-9155112578, Fax: +98-5138414499, E-mail: alamdarana@mums.ac.ir \\ Received 2016 September 09; Revised 2017 August 21; Accepted 2017 October 09.
}

\begin{abstract}
Background: Clinical diagnosis of malrotation is difficult to achieve, especially in older children and adults as its nonspecific presentations at this stage are usually neglected leading to delayed diagnosis of these cases.

objectives: In this study, we aimed to evaluate the diagnostic values of ultrasound and upper gastrointestinal (GI) series in patients with suspected intestinal malrotation.

Patients and Methods: This six-year cross-sectional, prospective study was conducted in Dr. Sheikh Children's Hospital affiliated to Mashhad University of Medical Sciences, Mashhad, Iran from 2009 to 2015. Totally, 67 children (aged < 15 years) with clinical findings of intestinal malrotation who had evidence of malrotation in ultrasound or upper GI series were enrolled in the study. Results of radiological evaluation were compared to the final diagnosis of patients achieved from surgery. Data analysis was performed using SPSS software.

Results: Of the 67 enrolled participants with clinical findings or suspected radiological signs of intestinal malrotation, about half (52.2\%) were male. Patients were 2 days to 16 years (mean, 2.5 years) old. Associated anomalies were observed in $47.7 \%$ of cases. Sensitivity and specificity were $82.5 \%$ and $85.7 \%$, respectively for GI series findings and $82.3 \%$ and $54.5 \%$, respectively for ultrasound compared with surgical findings as a gold standard.

Conclusion: Ultrasound has a similar diagnostic value compared to upper GI series. They are complementary examinations and negative ultrasound or GI series results do not necessarily rule out intestinal malrotation. Mesenteric vessels ultrasound could be used as more specific diagnostic method for the detection of intestinal rotational disorders.
\end{abstract}

Keywords: Intestinal Malrotation, Children, Ultrasound, GI series

\section{Background}

During normal fetal abdominal development, the three divisions of the GI tract (foregut, midgut, and hindgut) herniate out from the abdominal cavity. Afterwards, they undergo a $270^{\circ}$ counterclockwise rotation around the axis of the superior mesenteric vessel. Following this rotation, the bowels return to the abdominal cavity, which results in the fixation of the duodenojejunal junction to the left side of the midline and the placement of the cecum in the right lower quadrant.

Intestinal malrotation refers to any variation in the normal process of rotation and fixation of the GI tract. Complete arrest in the normal process of development (nonrotation) results in the placement of the duodenojejunal junction on the right side of the spinal column, while the total colon lies in the left abdomen. Malrotation is an incomplete bowel rotation that leads to abnor- mal position of the duodenojejunal junction and cecum. This wide range of abnormalities leads to various acute and chronic presentations of the disease $(1,2)$. Since this disease is often associated with acute symptoms such as acute midgut volvulus, catastrophic risk of ischemia, and midgut gangrene, it must be considered as a serious surgical emergency and must be immediately diagnosed and the patients should undergo surgery prior to development of midgut gangrene (3).

In a study performed in Hawaii, the occurrence of intestinal malrotation was reported to be 2.8 cases per 10,000 live births (4). Although intestinal malrotation usually occurs over the first months of life, it can present in later stages of life resulting in a more convoluted diagnostic process and its possible drawbacks $(5,6)$.

To prevent the loss of a long segment of the small intestine caused by midgut volvulus, malrotation should be immediately ruled out in any neonate with bilious vomiting, 
and urgent operative interventions must be carried out in case of any risk of volvulus.

Evaluation of the upper gastrointestinal series (UGI) is a diagnostic method for intestinal malrotation. However, it seems that this method is not accurate enough to detect malrotation and midgut volvulus. Nowadays, other diagnostic methods, such as ultrasonography and computed tomography (CT) scan are recommended for detection of intestinal malrotation $(7,8)$.

Although abdominal ultrasonography is suggested as a diagnostic screening test, contrast-enhanced imaging evaluations (preferably of the upper-gastrointestinal tract) must be performed in suspected cases to rule out malrotation (8). Other diagnostic methods, such as magnetic resonance imaging (MRI), which is used to detect malrotation or volvulus are not often suitable in acute cases (9).

\section{Objectives}

In this study, we aimed to evaluate the diagnostic values of abdominal ultrasonography and GI series in patients with suspected intestinal malrotation.

\section{Patients and Methods}

This six-year, prospective, cross-sectional study was conducted by two skilled pediatric radiologists on 67 children (aged $<15$ years) with clinical and radiographic findings of malrotation in Dr. Sheikh Children's hospital affiliated to Mashhad University of Medical Sciences, Mashhad, Iran from 2009 to 2015. Sixty-seven children underwent surgery, 54 had upper GI series, and ultrasound study was conducted in 55 patients.

In our hospital, ultrasound was the first diagnostic modality in the evaluation of abdominal complaints. If ultrasonographic findings of malrotation were observed and clinical findings were compatible with sonographic findings, especially in the presence of whirlpool sign and bilious vomiting, surgery was planned. As some cases with malrotation were asymptomatic or had vague complaints and inversion of the mesenteric vessels could be an incidental finding, upper GI series was performed in these cases to confirm the diagnosis. In few patients with suspected clinical symptoms and signs and normal ultrasound findings, upper GI series was also done.

Gray-scale ultrasonography was performed using sonographic 7.5 - $12 \mathrm{MHz}$ linear and 3.5 MHz curved probes. The subjects were scanned in the supine position with the transducer positioned in the midline of the anterior upper abdomen for detecting the location and direction of mesenteric vessels. Graded compression ultrasound from the sub-xiphoid to the umbilical region is necessary in almost all children which enables us to have a better visualization of the position of mesenteric vessels. So performing graded compression during inspiration and between two crying sounds (sobs) especially when the child is calm and relaxed is an important technique that should be taken into consideration in pediatric abdominal ultrasonography. Owing to this technique, ultrasound scanning takes less time than what is needed for upper GI series.

The superior mesenteric artery (SMA) was identified along with its echogenic walls, originating from the anterior wall of the aorta. The superior mesenteric vein (SMV) was localized by tracing the portal vein confluence and moving caudally until the direction of mesenteric vein was identified.

Sonographic findings of malrotation including inversion of mesenteric vessels, abnormal pathway of mesenteric vessels, duplication of the mesenteric vein and anterior position of the mesenteric vein relative to mesenteric artery were assessed (Figure 1).

Radiologic signs of malrotation such as abnormal location of ligamentum teres (duodenojejunal junction), and abnormal position of jejunum and cecum (Figure 2) were also evaluated and compared with clinical and surgical findings. Data analysis was performed using statistical package for the social science (SPSS) version 16. T-student test was used to compare quantitative variables such as age. Spearmen correlation test was assessed to find association between ultrasound and GI series results. P value less than 0.05 was considered as significant.

\section{Results}

Totally, among 67 evaluated children with clinical presentations and radiological signs of suspected intestinal malrotation, 35 (52.2\%) were male. The mean age of the participants was 2.5 years ranging from 2 days to 15 years. Forty-five $(67.2 \%)$ cases were younger than 2 years, eight (11.9\%) were between 2 and 5 years and fourteen (20.9\%) were older than 5 years. The mean age in patients diagnosed as malrotation by different methods is shown in Table 1 .

Table 1. Mean Age of Participants (Months) Diagnosed as Malrotation by Different Methods $^{\mathrm{a}}$

\begin{tabular}{lccc}
\hline Method of imaging & Malrotation & Other diagnoses & P value \\
\hline Abdominal ultrasound & $37.4 \pm 52.4$ & $17.9 \pm 32.9$ & 0.065 \\
Gastrointestinal series & $40.0 \pm 54.6$ & $24.3 \pm 36.3$ & 0.213 \\
\hline
\end{tabular}

${ }^{\mathrm{a}}$ Values are expressed as mean $\pm \mathrm{SD}$ 

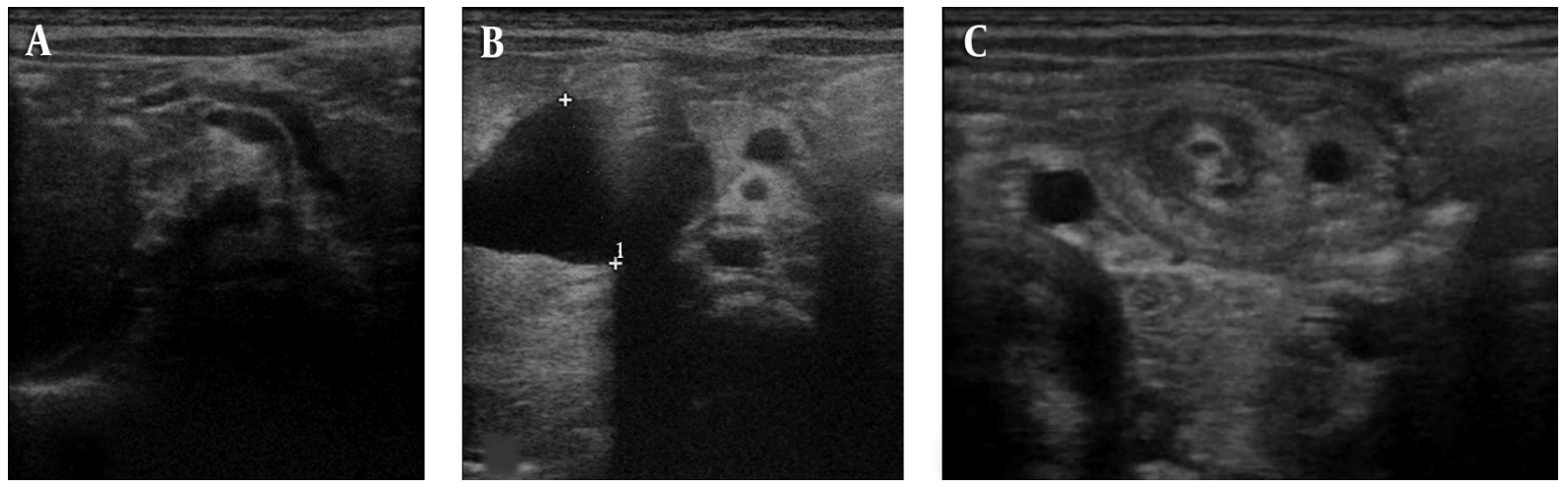

Figure 1. Gray-scale ultrasonography was performed, using sonographic 7.5 - $12 \mathrm{MHz}$ linear and $3.5 \mathrm{MHz}$ curved probes. The subjects were scanned in the supine position with the transducer positioned in the midline of the anterior upper abdomen. Graded compression sonography was performed for detecting the location and direction of mesenteric vessels. The superior mesenteric artery (SMA) was identified along with its echogenic walls, originating from the anterior wall of the aorta. The superior mesenteric vein (SMV) was localized by tracing the portal vein confluence and moving caudally until the direction of the mesenteric vein was identified. A, The abnormal pathway of mesenteric vessels toward front and above in right diaphragmatic hernia cases. B, The abnormal anterior position of the SMV and SMA in a patient with choledochal cyst. C, Left sided SMV and whirlpool sign in midgut volvulus.
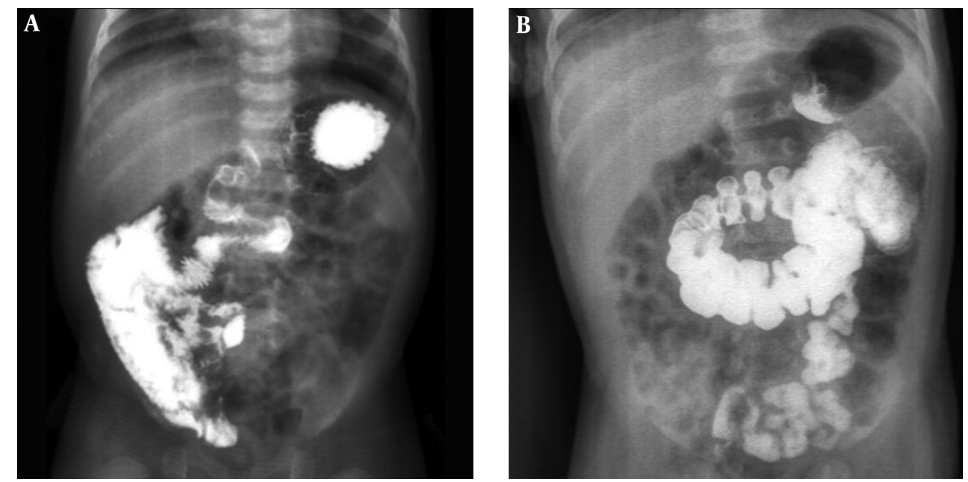

Figure 2. Radiologic findings of malrotation and midgut volvulus. A, Low location of duodenojejunal junction, corkscrew appearance of the duodenum and jejunum, and right side position of the jejunum. B, Abnormal position of the cecum below the transverse colon is seen.

No significant difference was observed in the mean age of patients with malrotation diagnosed with ultrasonography and those diagnosed by other methods $(\mathrm{P}=0.065)$. In addition, no significant difference was found between the mean age of patients with malrotation in GI series and children with other diagnoses $(\mathrm{P}=0.213)$. No significant difference was observed in the mean age of patients with final diagnosis of malrotation and those with other diagnoses $(\mathrm{P}=0.233)$.

After surgery, 57 patients (85\%) had the final diagnosis of malrotation. No sign of malrotation was observed in 10 patients in surgery. These 10 cases were normal or had other disorders, including annular pancreas, duodenal web, jejunal atresia, diaphragmatic hernia, adhesion and concomitant closed loop, internal hernia and choledochal cyst. Associated disorders were observed in half of the patients.
Among 67 subjects, volvulus was observed in six (8.9\%) patients. Whirlpool sign was found in five patients with volvulus resulting from malrotation and in one patient with adhesion related obstruction. Evaluation of the digestive system with GI series was performed in 54 children. Results obtained from UGI series and ultrasonography evaluation are presented in Table 2 .

Sensitivity and specificity of GI series results were $82.5 \%$ and $85.7 \%$, respectively. On the other hand, positive and negative predictive values of GI series evaluation were $97 \%$ and $46 \%$, respectively. Ultrasound sensitivity and specificity were $82.3 \%$ and 54.5\%, respectively. Moreover, positive and negative predictive values for ultrasound were $89.4 \%$ and $40 \%$, respectively. The correlation between ultrasound and GI series results is showed in Figure 3.

No significant correlation was observed between the results of ultrasound and UGI series $(r=0.095, P=0.495)$. 
Table 2. Results from GI Series and Abdominal Ultrasound Evaluation in the Detection of Rotational Anomaly in Children

\begin{tabular}{|ccc|}
\hline & \multicolumn{2}{c}{ Final diagnosis of the patients } \\
\cline { 2 - 3 } & Malrotation & Other diagnoses \\
\hline Gastrointestinal series & 33 & 1 \\
\hline $\begin{array}{l}\text { Presence of radiologic signs of } \\
\text { malrotation }\end{array}$ & 7 & 6 \\
\hline $\begin{array}{l}\text { Lack of radiologic signs of } \\
\text { malrotation }\end{array}$ & \multicolumn{2}{|c}{} \\
\hline $\begin{array}{l}\text { Ultrasound } \\
\text { Presence of ultrasound signs of } \\
\text { malrotation }\end{array}$ & 42 & 5 \\
\hline $\begin{array}{l}\text { Lack of ultrasound signs of } \\
\text { malrotation }\end{array}$ & 9 & 6 \\
\hline
\end{tabular}

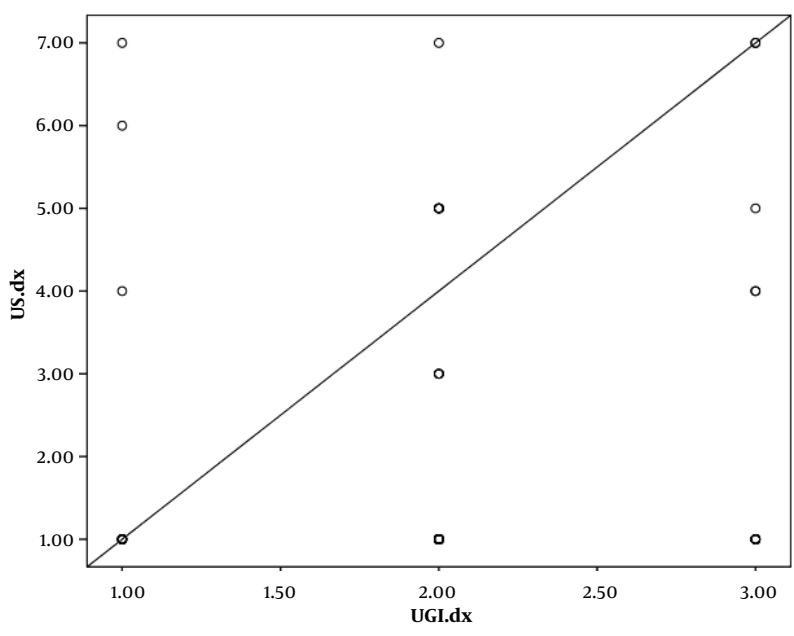

Figure 3. Correlation between the results of ultrasound and gastrointestinal series evaluation. upper gastrointestinal series (UGI).

Significant correlation was found between the results of UGI series evaluation and gender $(r=0.253, \mathrm{P}=0.039)$, while this association was not observed in the results obtained from ultrasound.

\section{Discussion}

Intestinal malrotation is a congenital anomaly that must be considered in all children at any age with symptoms of small intestinal obstruction or chronic abdominal pain, especially when they do not respond to treatment (8). To prevent acute or chronic and long-term complications such as volvulus and chronic intestinal obstruction in these patients, early and accurate diagnosis is mandatory (9-11).

In acute conditions, diagnostic methods such as CT scan or MRI, which are used sometimes to detect malrota- tion or volvulus, are not suitable as the first-line diagnostic method (12). It seems that ultrasonography, if performed by an experienced sonographer, is suitable for screening and ruling out other causes of vomiting, intestinal obstruction, abdominal pain, and malrotation.

In the majority of centers and studies, an integration of clinical and radiological findings is the diagnostic method of choice. The key finding is the abnormal location of the upper gastrointestinal tract found in urgent UGI fluoroscopy, which is performed at the presence of an experienced pediatric surgeon or radiologist (13).

The relationship between malrotation and other anomalies was demonstrated in the current study and some other studies $(14,15)$. In the present study, nearly half of the cases had malrotation-associated anomalies.

In a study conducted by Orzech et al. the sensitivity and specificity of ultrasound in the diagnosis of malrotation was reported as $86.5 \%$ and $74.7 \%$, respectively (6). In the current study, the final diagnosis of 57 cases (85\%) was malrotation. Ultrasound sensitivity in the diagnosis of malrotation was $82.3 \%$ and its specificity was $54.5 \%$. Positive and negative predictive values of ultrasonography were $89.4 \%$ and $40 \%$, respectively. According to the results, 10 patients had no sign of malrotation in surgery. These cases were normal or had other associated disorders. It was revealed that inversion of the mesenteric vessels is not a characteristic of rotational disorders and this finding could be observed in some normal cases and other intestinal anomalies including Bochdalek hernia, annular pancreas, jejunal atresia, choledochal cyst, duodenal web, internal hernia, adhesion and concomitant closed loop. So in patients with this finding in ultrasound and suspected clinical symptoms of malrotation, UGI series should be done as a confirmatory test. In our study, inversion of mesenteric vessels in ultrasound was the most common finding of malrotation.

The diagnostic value of whirlpool sign was significant in the detection of midgut volvulus in studies performed by Pracros and Chao $(7,8)$. This sign was not specific for rotational disorders and was found in one patient with adhesion and concomitant closed loop. In our study, among 67 samples, six (8.9\%) had volvulus. Whirlpool sign was noted in five patients with volvulus resulting from malrotation and in one patient with adhesion and concomitant obstruction. In addition, rotation of mesenteric vessels in all volvulus cases was clockwise.

In the present study, sensitivity of GI series results was $82.5 \%$ and the specificity was $85.7 \%$. Positive and negative predictive values of GI series evaluation were $97 \%$ and $46 \%$, respectively. However, this does not mean that negative results of GI series evaluation ruled out malrotation in the subjects.

In a study carried out by Nayak in 2014, GI series evalua- 
tion led to the diagnosis of malrotation in $78 \%$ of suspected premature neonates (16). In another study conducted in 2000 , false negative malrotation results were observed in $15 \%$ of the cases using GI series evaluation (17). In a former study, contrast-enhanced evaluation of the upper gastrointestinal system detected malrotation in $41 \%$ of the patients, while assessment with barium enema led to detection of this diagnose in $34 \%$ of the patients. In another study performed in 2008 in America, although high sensitivity of GI series evaluation was confirmed in the detection of intestinal malrotation, this method did not have proper specificity and diagnostic value (18). In a study performed by Torres et al. 95\% sensitivity and $86 \%$ specificity was reported for upper GI series in malrotation diagnosis (19).

Table 3 shows sensitivity and specificity of upper GI series and ultrasound in some studies. Little discrepancy might be due to different sample sizes in the mentioned studies.

Table 3. Comparison of Sensitivity and Specificity of Upper Gastrointestinal (GI) Se ries and Ultrasound in Different Studies ${ }^{\mathrm{a}}$

\begin{tabular}{lcccc}
\hline \multirow{2}{*}{ Studies/Ref } & \multicolumn{2}{c}{ Upper GI Series } & \multicolumn{2}{c}{ Ultrasound } \\
\cline { 2 - 5 } & Sensitivity & Specificity & Sensitivity & Specificity \\
\hline $\begin{array}{l}\text { Torres et al. } \\
(\mathbf{1 9 )})\end{array}$ & 95 & 86 & - & - \\
$\begin{array}{l}\text { Sizemore et } \\
\text { al. (18) }\end{array}$ & 96 & - & - & - \\
$\begin{array}{l}\text { Nayak et al. } \\
(16)\end{array}$ & 78 & - & - & - \\
$\begin{array}{l}\text { Orzech et al. } \\
(6)\end{array}$ & & & & \\
\hline \begin{tabular}{l} 
Current study \\
\hline
\end{tabular} & 82.5 & 85.7 & 86.5 & 74.7 \\
\hline
\end{tabular}

${ }^{\mathrm{a}}$ Values are expressed as \%

It seems that the most important limitation of GI series was lack of accurate evaluation of duodenojejunal junction on first pass of barium from C loop of the duodenum. Diagnostic accuracy of GI series was improved in patients with normal variations of jejunum and duodenum $(20,21)$.

According to the results of the current study, sensitivity and specificity of ultrasound were higher in the detection of malrotation and midgut volvulus, compared to GI series. Our results show that ultrasound has a similar accuracy compared to GI series and considerably, it avoids exposure to ionizing radiation. High sensitivity of ultrasonography in the diagnosis of malrotation and midgut volvulus in our study and other similar studies reveals that these critical cases can be easily diagnosed with ultrasonography. Attention to normal variations of mesenteric vessels without a doubt increased the specificity of ultrasound examination.

Normal variations in mesenteric vessels such as ante- rior location of the cranial part of superior mesenteric vein (SMV) relative to SMA and duplication of SMV in the cranial part and abnormal pathway of mesenteric vessels should be considered to prevent over diagnosis of malrotation (22).

Lack of radiation exposure as well as convenience of performing ultrasonography at the patient's bed makes it the most accurate method for diagnosis of intestinal malrotation, though evaluation of mesenteric vessels is recommended as an essential component of abdominal ultrasonography in children.

In conclusion, ultrasound has a similar diagnostic value compared to upper GI series. They are complementary examinations and negative ultrasound or GI series alone does not necessarily rule out intestinal rotation. Mesenteric vessel ultrasonography could be used as more specific diagnostic method for the detection of intestinal rotational disorders.

\section{Acknowledgments}

We express our appreciation to the radiology technicians of Dr. Sheikh Children's hospital who provided technical support that greatly assisted the research.

\section{Footnote}

Financial Disclosure: Dr. Mina Taghavi and Dr. Ali Alamdaran received research funding from Dr. Sheikh Children's Hospital. The terms of this arrangement have been reviewed and approved by Mashhad University of Medical Sciences in accordance with its conflict of interest policies.

\section{References}

1. Ballesteros Gomiz E, Torremade Ayats A, Duran Feliubadalo C, Martin Martinez C, Caro Tarrago A. Intestinal malrotation-volvulus: imaging findings. Radiologia. 2015;57(1):9-21. doi: 10.1016/j.rx.2014.07.007. [PubMed: 25458122].

2. Moldrem AW, Papaconstantinou H, Broker H, Megison S, Jeyarajah DR. Late presentation of intestinal malrotation: an argument for elective repair. World JSurg. 2008;32(7):1426-31. doi:10.1007/s00268-008-94903. [PubMed: 18347850$]$.

3. Drewett M, Johal N, Keys C, J. Hall N, Burge D. The burden of excluding malrotation in term neonates with bile stained vomiting. Pediatr Surg Int. 2016;32(5):483-6. doi: 10.1007/s00383-016-3877-2. [PubMed: 26895031].

4. Cullis PS, Siminas S, Losty PD. Is Screening of Intestinal Foregut Anatomy in Heterotaxy Patients Really Necessary?: A Systematic Review in Search of the Evidence. Ann Surg. 2016;264(6):1156-61. doi: 10.1097/SLA.0000000000001563. [PubMed: 26704743].

5. Bicer S, Celik A. Duodenal Obstruction Caused by Acute Appendicitis with Intestinal Malrotation in a Child. Am J Case Rep. 2015;16:574-6. doi: 10.12659/AJCR.894311. [PubMed: 26317163]. 
6. Orzech N, Navarro OM, Langer JC. Is ultrasonography a good screening test for intestinal malrotation? J Pediatr Surg. 2006;41(5):1005-9. doi: 10.1016/j.jpedsurg.2005.12.070. [PubMed: 16677901].

7. Raitio A, Green PA, Fawkner-Corbett DW, Wilkinson DJ, Baillie CT. Malrotation: Age-Related Differences in Reoperation Rate. Eur J Pediatr Surg. 2016;26(1):34-7. doi: 10.1055/s-0035-1563677. [PubMed: 26394370].

8. Heidsma CM, Hulsker CC, van der Zee D, Kramer WH. [Malrotation with or without volvulus]. Ned Tijdschr Geneeskd. 2015;159. A8859. [PubMed: 26173661].

9. Dufour D, Delaet MH, Dassonville M, Cadranel S, Perlmutter N. Midgut malrotation, the reliability of sonographic diagnosis. Pediatr Radiol. 1992;22(1):21-3. [PubMed: 1594305].

10. Strouse PJ. Clinics in diagnostic imaging (74). Midgut malrotation with volvulus. Singapore Med J. 2002;43(6):325-8. [PubMed: 12380734].

11. Gunsar C, Karaca I, Sencan A. Clinical presentation and radiographic identification of intestinal rotation anomalies in symptomatic neonates. Pediatr Cerrahi Derg. 2002;16(1):12-5.

12. Forrester MB, Merz RD. Epidemiology of intestinal malrotation, Hawaii, 1986-99. Paediatr Perinat Epidemiol. 2003;17(2):195-200. [PubMed: 12675787].

13. Berdon WE. The diagnosis of malrotation and volvulus in the older child and adult: a trap for radiologists. Pediatr Radiol. 1995;25(2):1013. [PubMed: 7596651].

14. Weinberger E, Winters WD, Liddell RM, Rosenbaum DM, Krauter D. Sonographic diagnosis of intestinal malrotation in infants: importance of the relative positions of the superior mesenteric vein and artery. AJR Am J Roentgenol. 1992;159(4):825-8. doi: 10.2214/ajr.159.4.1529850. [PubMed: 1529850].
15. Nahvi H, Ahmadi J, Kalantari M, Molaeian M, Sedighi A, Poorang H, et al. Congenital intestinal malrotation as the serious cause of neonatal intestinal obstruction. Iran J Pediatr. 2005;15(3):215-20.

16. Nayak GK, Levin TL, Kurian J, Kohli A, Borenstein SH, Goldman HS. Bedside upper gastrointestinal series in critically ill low birth weight infants. Pediatr Radiol. 2014;44(10):1252-7. doi: 10.1007/s00247-014-29931. [PubMed: 24805204].

17. Dilley AV, Pereira J, Shi EC, Adams S, Kern IB, Currie B, et al. The radiologist says malrotation: does the surgeon operate? Pediatr Surg Int. 2000;16(1-2):45-9. [PubMed: 10663834].

18. Sizemore AW, Rabbani KZ, Ladd A, Applegate KE. Diagnostic performance of the upper gastrointestinal series in the evaluation of children with clinically suspected malrotation. Pediatr Radiol. 2008;38(5):518-28. doi: 10.1007/s00247-008-0762-8. [PubMed: 18265969].

19. Torres AM, Ziegler MM. Malrotation of the intestine. World J Surg. 1993;17(3):326-31. [PubMed: 8337878].

20. Long FR, Kramer SS, Markowitz RI, Taylor GE, Liacouras CA. Intestinal malrotation in children: tutorial on radiographic diagnosis in difficult cases. Radiology. 1996;198(3):775-80. doi: 10.1148/radiology.198.3.8628870. [PubMed: 8628870].

21. Lim-Dunham JE, Ben-Ami T, Yousefzadeh DK. Manual epigastric compression during upper gastrointestinal examination of neonates: value in diagnosis of intestinal malrotation and volvulus. AJR Am J Roentgenol.1999;173(4):979-83. doi: 10.2214/ajr.173.4.10511161. [PubMed: 10511161].

22. Alamdaran SA, Hashemi J, Layegh P, Taghawi M. The Diagnostic Value of Ultrasonography in Pediatric Intestinal Malrotation. Growth. 2015;5:8.3. 